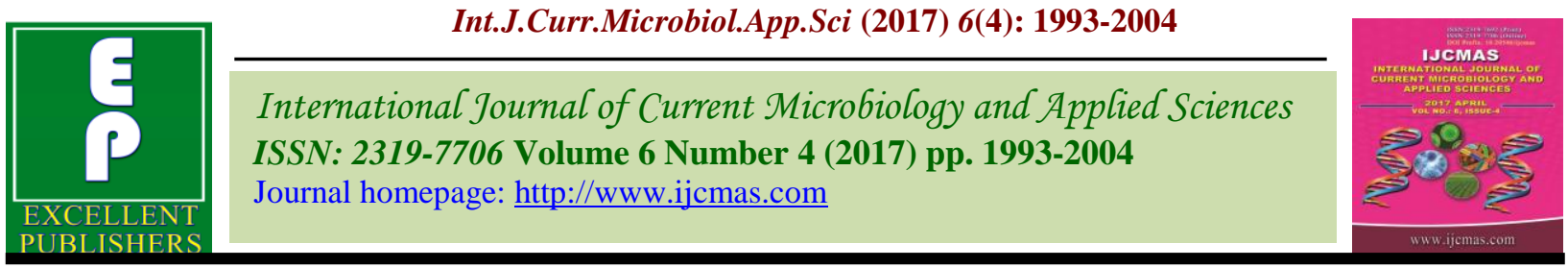

Original Research Article

https://doi.org/10.20546/ijcmas.2017.604.237

\title{
Physiological Basis of Water Logging Tolerance in Wheat at Vegetative Stage Under Sodic Soil
}

\author{
Ompal Singh $^{1 *}$, S.P. Singh ${ }^{1}$, Raj Bahadur ${ }^{1}$, Mani Ram ${ }^{1}$, \\ Prashansha Singh $^{1}$ and Sunil Prajapati ${ }^{2}$
}

\author{
${ }^{1}$ Department of Plant Physiology, N. D. University of Agriculture and Technology, \\ Faizabad 224 229, Uttar Pradesh, India \\ ${ }^{2}$ Department of Horticulture, Jawaharlal Nehru Krishi Vishwa Vidyalaya, \\ Jabalpur 482004, Madhya Pradesh, India \\ *Corresponding author
}

\section{A B S T R A C T}

\section{Keywords}

Wheat, Water logging, Sodic soil, KRL 3-4, KRL 99, HD 2009, HD 2851.

Article Info

Accepted:

15 March 2017

Available Online:

10 April 2017
The present field investigation resulted; highest plant height was recorded in KRL 99 while minimum in HD 2851. While less in tolerant varieties; KRL 3-4 and KRL 99 at 40 and 80 DAS. Water logging induced more reduction in tiller in susceptible variety HD 2009 followed by HD 2851 and very less reduction in tolerant varieties KRL 3-4 and KRL 99 at 40 and 80 DAS. The biomass plant ${ }^{-1}$ was more depleted in wheat susceptible varieties HD 2009 and HD 2851, while minimum in KRL 99 followed by KRL 3-4 at 40,80 DAS. Chlorophyll content extent of decrease was less in KRL 3-4 and KRL 99 as compared to HD 2851 and HD 2009. Higher soluble carbohydrate was recorded in HD 2009 and HD 2851, higher starch content was recorded in KRL 99 and KRL 3-4 and lower in HD 2009 at 40, 80 DAS respectively, maximum Na content was estimated in HD 2009, while minimum in KRL 99 in comparison to control. Highest K in KRL 3-4 while lowest in HD 2009 while higher Ca in KRL 99 and lowest in HD 2851. Maximum Fe content in HD 2009 while minimum in KRL 99. Maximum Al and Mg content were in HD 2009, while minimum in KRL 3-4. Maximum number of ear plant ${ }^{-1}$ and number of grains ear was observed in HD 2009 and HD 2851, while minimum in KRL 99. Water logging produced higher reduction in HD 2009 followed by HD 2851, while lowest in KRL 3-4. Higher biomass and test weight in HD 2009, while lowest in KRL 3-4. Highest harvest index was recorded in KRL 99, while lowest in HD 2009.

\section{Introduction}

Wheat (Triticum aestivum L.) is one of the most important cereal staple food crops of the world. Salinity, alkalinity and water logging are the major stresses restricting crop stand and yield. Water logging is a serious problem affecting crop growth and productivity. Large areas of water logging occur in the irrigated rice-wheat rotation system used throughout South and South East Asia. Wheat is experiences water logging in these areas due to sub soil compaction during puddling for rice planting (Samad et al., 2001), though the individual effects of water logging, salinity and alkalinity on germination, seedling growth and physiological processes and the combined effects of salinity and water logging on wheat and other crops are reported by (Dong et al., 1998 and Sharma et al., 2005 
b). Very little information is available on the combined effects of the alkalinity and water logging stress (Sharma et al., 2004). Some waterlogged soils become rich in $\mathrm{Mn}^{+2}$ and $\mathrm{Fe}^{+2}$, devoid of $\mathrm{NO}_{3}{ }^{-}$and $\mathrm{SO}_{4}^{-2}$ and anaerobic microbial metabolites may accumulate. These effects become more pronounced during prolonged periods of water logging.

Water logging occurs in many wheat growing regions across the world. According to an estimate about10-15 Mha of world's wheat growing areas are affected by water logging each year (Sayra et al., 1994). It represents 15-20\% of the70 Mha sown to wheat each year. Water logging problem for wheat production is more acute especially on sodic or alkaline soils of India. Long term surveys indicate that every time a Sodic soil is irrigated in India, it is usually water logged, these soils become anoxic with redox potential $350 \mathrm{mV}$ (Setter et al., 2006). In India, 2.5 million ha of alkaline soils of IndoGangetic plains planted with wheat may experience saturated or temporary waterlogged conditions every year due to excess rains or mismanagement of water drainage from the farmer's field (Sharma et al., 1998).

Crop losses depend on the stages of growth at the time of water logging and it may range from few per cent to complete crop failure if water logging occurs during very early vegetative growth. Water logging tolerance of wheat may differ depending upon the stage of growth and other environmental factors. Variable results have been reported for yield loss versus stage of water logging. Watson $e t$ al., (1976) reported larger reduction in grain yield of wheat, barley and oat when 6 weeks of continuous water logging started at 2 weeks after sowing in comparison to water logging started at 6 weeks or 10-14 weeks (ear emergence) after sowing. However, Gardner and Flood (1993) suggested that early reproductive stage are more adversely affected by water logging than tillering stage due to reduction in grains ear ${ }^{-1}$. Bao (1997) found that for twenty varieties of wheat, the order of tolerance to water logging at different stages was booting > jointing > tillering > grain filling.

The lack of knowledge about key traits in field environments is a major constraint to germplasm improvement and crop management because water logging-prone environments are highly diverse and complex, and the mechanisms of tolerance to water logging include a large range of traits. This is further evaluated with the aim of prioritizing traits required for water logging tolerance of wheat in the field. Keeping in view of above facts the present investigation was undertaken to differential response of wheat varieties to water logging so that we can be used as parents for future germplasm improvement in wheat of water logging tolerance under sodic soil conditions.

\section{Materials and Methods}

The present investigation was conducted during Rabi season 2011-12, at Department of Crop Physiology, Narendra Deva University of Agriculture and Technology, Kumarganj, Faizabad (U.P.). The experiment was conducted in earthen pots containing sodic soil with two genotypes KRL 99, KRL 3-4 (tolerant) and HD2009, HD 2851 (susceptible); the whole experiment was planned under Complete Randomized Block Design (CRBD) with three replications along with eight treatments. Faizabad district falls in semi-arid zone, receiving a mean annual rainfall of $1100 \mathrm{~mm}$, of which about $80 \%$ of total precipitation occurs during monsoon season, and lies between a latitude $24.47^{\circ}$ and $26.56^{\circ}$ North and longitude of 81.12 and $83.90^{\circ}$ East on an elevation of about 113 meters above mean sea level. The physical 
and chemical properties of the experimental soil are as fallow; Soil $\mathrm{pH}$ : 8.8-9.1, TextureSalty loam, Organic carbon- $0.32 \%$, Available NPK- (106.0: 13.52: $230.50 \mathrm{~kg}$ ha ${ }^{1}$ ), NPK were added at the rate of 120:60:60 $\mathrm{kg} \mathrm{ha}^{-1}$, each pot was lined with polythene bag and filled with $7 \mathrm{~kg}$ well pulverized sodic soil collected from field of MES, Kumarganj. Fifteen bold and healthy seeds were sown in pots at $2 \mathrm{~cm}$ depth on 24 December 2011.

All the observations were recorded at three stages of crop growth i.e. $40^{\text {th }}, 80^{\text {th }}$ at maturity days after sowing. Three plants under each treatment were tagged initially which were used for growth parameters viz., Plant height $(\mathrm{cm})$, Biomass plant $^{-1}(\mathrm{~g}), 50 \%$ flowering, Maturity duration and yield and yield attributes traits viz., Number of ears plant ${ }^{-1}$, Ear length per plant ${ }^{-1}$, Number of grains plant ${ }^{1}$, Test weight, Grain yield plant ${ }^{-1}$, Harvest index, other bio-chemical parameters as; Chlorophyll content; Total Chlorophyll $=20.2$ x O.D. (645) + 8.02 x O.D. (663) X (V/1000 x $\mathrm{W})$, Total soluble carbohydrate $\mathrm{mg} / \mathrm{g}$ (Yemmi and Willis 1954), Starch content (Mc Cready et al., 1950), Mineral analysis- The $\mathrm{Fe}, \mathrm{Al}$ and $\mathrm{Mn}$ were analyzed with the help of atomic absorption spectrophotometer. The statistical analysis of data was done by the method described by Panse and Sukhatme (1978) using completely randomized design (CRD) factorial experiment.

\section{Results and Discussion}

\section{Germination and growth parameters}

The highest plant height was recorded in KRL 99 (Table 1a) while minimum height was observed in HD 2851 at different growth stages. However, extent of decrease was less in tolerant varieties; KRL 3-4 (11.0 and $10.4 \%)$ and KRL 99 (9.9 and 10.9\%) as compared to susceptible varieties; HD2851 (28.6 and 29.9) and HD 2009 (19.9 and 20.0) at 40 and 80 DAS. Among tolerant varieties less reduction was noted in case of KRL 99 while susceptible HD 2851 varieties showed less reduction than the other varieties HD 2009. Mean effect of all varieties at different stages registered significant variation in total plant height among varieties. Mean effect of water logging treatment showed significant decrease in plant height under water logging over non water logging. The perusal of data indicates that plant height significantly reduced at maturity stage due to water logging treatments imposed at vegetative stage. KRL 3-4 showed significantly greater plant height over rest of the varieties. Susceptible varieties HD 2009 showed maximum reduction (29.2) while minimum was in tolerant variety KRL 3-4 (11.7) under water logging at vegetative stages, so vegetative stage was most sensitive to water logging.

Number of tillers plant ${ }^{-1}$ (Table 1b) decreased under water logging relative to non water logging in all the varieties, tolerant varieties KRL 99 had maintained more tillers plant ${ }^{-1}$ while lowest number was observed in susceptible variety HD 2009. Water logging induced more reduction in tiller in susceptible variety HD 2009 (35.5, 33.5 and $33.5 \%)$ followed by HD 2851 (31.0, 28.2 and 28.2\%) at 40,80 and maturity respectively. While registered very less reduction tolerant varieties KRL 3-4(19.9, 15.4 and 15.4\%) and KRL $99(18.9,16.7$ and $16.7 \%)$ at 40 and 80 DAS and maturity in under water logging over non water logging. The data reveal that higher biomass plant ${ }^{-1}$ was obtained in tolerant variety KRL 99 and KRL 3-4 while lower biomass plant $^{-1}$ was observed in susceptible varieties HD 2009 and HD 2851 at 40, 80 days and maturity after sowing.

The biomass plant ${ }^{-1}$ was more depleted in wheat susceptible varieties HD 2009 (59.7, 42.0 and $47.3 \%)$ and HD 2851 (40.9, 32.5 and $35.8 \%$ ), while minimum depletion was in 
tolerant varieties KRL 99 (25.2, 13.3 and 20.6 $\%)$ followed by KRL 3-4 (23.3, 15.7 and $20.0 \%$ ) at 40,80 DAS and maturity. $50 \%$ flowering was observed in susceptible varieties HD 2851 and HD 2009 (1-6 days) while minimum delay was in tolerant varieties KRL 99 and KRL 3-4 (1-4 days) Maturity duration too was delayed by water logging stress, the maximum delayed of susceptible varieties HD 2851 and HD 2009(1-5) days was observed in KRL 99 and KRL 3-4. Similar results have also been reported by Sharma et al., (2005 a) in pigeon pea, Savita et al., (2004) in maize, Srivastava et al., (2004), Neog et al., (2002) in rice and Baranwal and Singh (2002) in maize, Lin Ji Xiang et al., (2012).

\section{Biochemical parameters}

Chlorophyll content (Table 2) decreased under water logging relative to non water logging in all the varieties, however, extent of decrease was less in tolerant varieties; KRL 34 and KRL99 (6-10 and 3-7\%) as compared to susceptible varieties; HD 2851 and HD 2009(14.20 and 6-7\%) at both the stages of observation 40 and 80 DAS. Among the tolerant varieties, KRL-99 had less decrease in chlorophyll than KRL 3-4, while among susceptible HD 2009 showed highest reduction. Similar findings were also reported by Sharma et al., (2005 b) in wheat, Das and Sarkar (2003) in rice, Baranwal and Singh (2002) in maize and Neog et al., (2002) in rice, Prasad et al., (2004), Singh and Sharma (2010).

Soluble carbohydrate in leaf generally increased with increasing plant age in all the wheat varieties. Higher soluble carbohydrate was recorded in wheat tolerant variety KRL 99 and KRL 3-4 and lower in susceptible varieties HD 2009 and HD 2851 at both the stages of observation. However, the reduction in soluble carbohydrate under water logging was more in susceptible varieties HD 2009(38.2 and 25.7\%) and HD 2851 (29.5 and $20.7 \%$ ) than tolerant varieties KRL 99 (28.8 and 18.0\%) and KRL 3-4 (20.5 and $12.6 \%$ ) at 40 and 80 DAS found respectively. Similar views have been advocated by Sharma et al., (2005 b) in wheat, Singh et al., (2005) in Rice, Prasad et al., (2004), Rai et al., (2004), Baranwal and Singh (2002) in maize and Ram et al., (2002) in low land rice. Similar results have also been reported by Pattanagul and Thitisaksakul (2008).

Soluble starch content in leaf generally increased with increasing plant age in all the wheat varieties. Higher starch content was recorded in wheat tolerant variety KRL 99 and KRL 3-4 and lower in susceptible varieties HD 2009 (26.5, 20.2\%) and HD $2851(24.0,20.2 \%)$ at both 40 and 80 DAS. However, the reduction in starch content under water logging was more in susceptible varieties HD 2009 and HD 2851 at 40 and 80 days than tolerant varieties KRL 99 (19.3,14.8\%) and KRL 3-4 (12.2 and 10.5\%) at 40 and 80 DAS respectively.

\section{Yield and yield attributes}

Number of ear plant ${ }^{-1}$ (Table 3) decreased significantly under water logging relative to non water logging in all the varieties. The maximum number of ear plant ${ }^{-1}$ was recorded in wheat variety KRL 99 which was at par with KRL 3-4 and significantly superior over rest of the varieties while minimum in HD 2009 under the water logging treatment. Maximum reduction under WL treatment relative to control was observed in susceptible varieties HD 2009 (32.5\%) and HD 2851 $(29.8 \%)$ than tolerant varieties KRL 99 (19.7\%) and KRL 3-4(19.7\%). Mean effect of water logging treatment showed non significant decrease in starch content over non water logging. 


\section{Int.J.Curr.Microbiol.App.Sci (2017) 6(4): 1993-2004}

Table.1 (a) Effect of water logging on Germination and growth parameters of wheat varieties under sodic soil

\begin{tabular}{|c|c|c|c|c|c|c|c|c|c|c|c|c|c|c|c|c|c|c|}
\hline \multirow{3}{*}{ Varieties } & \multicolumn{9}{|c|}{ Plant height (cm) } & \multicolumn{9}{|c|}{ Number of tillers plant ${ }^{-1}$} \\
\hline & \multicolumn{3}{|c|}{40 DAS } & \multicolumn{3}{|c|}{80 DAS } & \multicolumn{3}{|c|}{ At maturity } & \multicolumn{3}{|c|}{40 DAS } & \multicolumn{3}{|c|}{80 DAS } & \multicolumn{3}{|c|}{ At maturity } \\
\hline & NWL & WL & Mean & NWL & WL & Mean & NWL & WL & Mean & NWL & WL & Mean & NWL & WL & Mean & NWL & WL & Mean \\
\hline $\begin{array}{l}\text { KRL- 3-4 } \\
\text { (T) }\end{array}$ & 35.60 & $\begin{array}{c}31.68 \\
(11.01)\end{array}$ & 33.64 & 77.34 & $\begin{array}{c}69.30 \\
(10.40)\end{array}$ & 73.32 & 77.82 & $\begin{array}{c}68.71 \\
(11.71)\end{array}$ & 73.27 & 2.21 & $\begin{array}{c}1.77 \\
(19.91)\end{array}$ & 1.99 & 2.33 & $\begin{array}{c}1.97 \\
(15.45)\end{array}$ & 2.15 & 2.33 & $\begin{array}{c}1.97 \\
(15.45)\end{array}$ & 2.15 \\
\hline KRL -99 (T) & 37.05 & $\begin{array}{l}33.38 \\
(9.91)\end{array}$ & 35.22 & 58.17 & $\begin{array}{c}51.83 \\
(10.90)\end{array}$ & 55.00 & 52.04 & $\begin{array}{c}58.94 \\
(13.26)\end{array}$ & 55.49 & 2.67 & $\begin{array}{c}2.17 \\
(18.73)\end{array}$ & 2.42 & 3.00 & $\begin{array}{c}2.50 \\
(16.67)\end{array}$ & 2.75 & 3.00 & $\begin{array}{c}2.50 \\
(16.67)\end{array}$ & 2.75 \\
\hline HD-2851 (S) & 35.26 & $\begin{array}{c}28.24 \\
(19.91)\end{array}$ & 31.75 & 55.78 & $\begin{array}{c}44.62 \\
(20.00)\end{array}$ & 50.20 & 55.99 & $\begin{array}{c}44.57 \\
(20.40)\end{array}$ & 50.28 & 2.00 & $\begin{array}{c}1.38 \\
(31.00)\end{array}$ & 1.69 & 2.13 & $\begin{array}{c}1.53 \\
(28.17)\end{array}$ & 1.83 & 2.13 & $\begin{array}{c}1.53 \\
(28.17)\end{array}$ & 1.83 \\
\hline HD-2009 (S) & 33.33 & $\begin{array}{c}23.80 \\
(28.59)\end{array}$ & 28.57 & 60.67 & $\begin{array}{c}42.53 \\
(29.90)\end{array}$ & 51.60 & 61.10 & $\begin{array}{c}43.22 \\
(29.26)\end{array}$ & 52.16 & 2.00 & $\begin{array}{c}1.31 \\
(34.50)\end{array}$ & 1.66 & 2.00 & $\begin{array}{c}1.33 \\
(33.50)\end{array}$ & 1.67 & 2.00 & $\begin{array}{c}1.33 \\
(33.50)\end{array}$ & 1.67 \\
\hline \multirow[t]{2}{*}{ Mean } & 35.31 & 29.28 & _- & 62.99 & $\mathbf{5 2 . 0 7}$ & _- & 61.74 & 53.86 & - & 2.22 & 1.66 & _- & 2.37 & 1.83 & _ & 2.37 & 1.83 & - \\
\hline & $\mathbf{V}$ & WL & VxWL & $\mathbf{V}$ & WL & VxWL & $\mathbf{V}$ & WL & VxWL & $\mathbf{v}$ & WL & VxWL & $\mathbf{V}$ & WL & VxWL & $\mathbf{V}$ & WL & VxWL \\
\hline $\operatorname{SEm} \pm$ & 0.66 & 0.47 & 0.94 & 1.20 & 0.85 & 1.70 & 1.21 & 0.85 & 1.70 & 0.04 & 0.03 & 0.05 & 0.04 & 0.03 & 0.06 & 0.04 & 0.03 & 0.06 \\
\hline CD at $5 \%$ & 1.98 & 1.40 & 2.81 & 3.61 & 2.55 & 5.10 & 3.61 & 2.56 & 5.11 & 0.12 & 0.08 & NS & 0.13 & 0.09 & NS & 0.13 & 0.09 & NS \\
\hline
\end{tabular}


Table.1 (b) Effect of water logging on Germination and growth parameters of wheat varieties under sodic soil

\begin{tabular}{|c|c|c|c|c|c|c|c|c|c|c|c|c|c|}
\hline \multirow[t]{3}{*}{ Varieties } & \multicolumn{9}{|c|}{ biomass plant $^{-1}(\mathrm{~g})$} & \multicolumn{2}{|c|}{$\begin{array}{l}\text { Days to } 50 \% \\
\text { flowering }\end{array}$} & \multicolumn{2}{|c|}{ Days to maturity } \\
\hline & \multicolumn{3}{|c|}{40 DAS } & \multicolumn{3}{|c|}{80 DAS } & \multicolumn{3}{|c|}{ At maturity } & \multirow[b]{2}{*}{ NWL WL } & \multirow[b]{2}{*}{ Mean } & \multirow[b]{2}{*}{ NWL } & \multirow[b]{2}{*}{ WL Mean } \\
\hline & NWL & WL & Mean & NWL & WL & Mean & NWL & WL & Mean & & & & \\
\hline $\begin{array}{l}\text { KRL- 3- } \\
4 \text { (T) }\end{array}$ & 1.16 & $\begin{array}{c}0.89 \\
(23.27)\end{array}$ & 1.03 & 3.50 & $\begin{array}{c}2.95 \\
(15.71)\end{array}$ & 3.22 & 4.00 & $\begin{array}{c}3.20 \\
(20.00)\end{array}$ & 3.60 & 75.0079.00 & 77.00 & 114.001 & 116.00115 .00 \\
\hline $\begin{array}{l}\text { KRL -99 } \\
\text { (T) }\end{array}$ & 1.51 & $\begin{array}{c}1.13 \\
(25.17)\end{array}$ & 1.32 & 3.60 & $\begin{array}{c}3.12 \\
(13.33)\end{array}$ & 3.36 & 4.27 & $\begin{array}{c}3.39 \\
(20.61)\end{array}$ & 3.83 & 71.0075.00 & 73.00 & 110.001 & 112.00111.00 \\
\hline $\begin{array}{l}\text { HD-2851 } \\
\text { (S) }\end{array}$ & 1.32 & $\begin{array}{c}0.78 \\
(40.91)\end{array}$ & 1.05 & 3.42 & $\begin{array}{c}2.31 \\
(32.46)\end{array}$ & 2.87 & 4.05 & $\begin{array}{c}2.60 \\
(35.80)\end{array}$ & 3.32 & 69.0074 .00 & 71.501 & 114.001 & 117.00115 .50 \\
\hline $\begin{array}{l}\text { HD-2009 } \\
\text { (S) }\end{array}$ & 1.24 & $\begin{array}{c}0.50 \\
(59.68)\end{array}$ & 0.87 & 3.24 & $\begin{array}{l}1.87 \\
(41.98)\end{array}$ & 2.56 & 3.78 & $\begin{array}{c}1.99 \\
(47.35)\end{array}$ & 2.88 & 70.0076 .00 & 73.001 & 113.001 & 118.00115 .50 \\
\hline \multirow[t]{2}{*}{ Mean } & 1.31 & 0.83 & - & 3.44 & 2.56 & - & 4.02 & 2.79 & - & 71.2576.00 & -1 & 112.751 & 115.75 \\
\hline & $\mathbf{v}$ & WL & VxWL & $\mathbf{v}$ & WL & VxWL & $\mathbf{V}$ & WL & VxWL & V WL & VxWL & $\mathbf{V}$ & WL VXWL \\
\hline $\mathrm{SEm} \pm$ & 0.03 & 0.02 & 0.04 & 0.06 & 0.04 & 0.08 & 0.07 & 0.05 & 0.10 & 1.831 .29 & 2.58 & 2.35 & $1.66 \quad 3.33$ \\
\hline $\begin{array}{l}\text { CD at } \\
5 \%\end{array}$ & 0.08 & 0.05 & 0.11 & 0.17 & 0.12 & 0.25 & 0.21 & 0.15 & 0.30 & $5.47 \quad 3.87$ & NS & NS & NS \\
\hline
\end{tabular}


Int.J.Curr.Microbiol.App.Sci (2017) 6(4): 1993-2004

Table.2 Effect of water logging on Biochemical parameters of wheat varieties under sodic soil

\begin{tabular}{|c|c|c|c|c|c|c|c|c|c|c|c|c|c|c|c|c|c|c|}
\hline \multirow[t]{3}{*}{ Varieties } & \multicolumn{6}{|c|}{$\begin{array}{l}\text { Total soluble carbohydrate content in leaf } \\
\text { ( } \mathrm{mg} \mathrm{g}^{-1} \text { dry wt.) }\end{array}$} & \multicolumn{6}{|c|}{ Starch content in leaf ( $\mathrm{mg} \mathrm{g}^{-1} \mathrm{dry}$ wt.) } & \multicolumn{6}{|c|}{$\begin{array}{c}\text { Total chlorophyll content ( } \mathrm{mg} \mathrm{g}^{-1} \text { fresh weight of } \\
\text { leaves) }\end{array}$} \\
\hline & \multicolumn{3}{|c|}{80 DAS } & \multicolumn{3}{|c|}{40 DAS } & \multicolumn{3}{|c|}{40 DAS } & \multicolumn{3}{|c|}{40 DAS } & \multicolumn{3}{|c|}{40 DAS } & \multicolumn{3}{|c|}{80 DAS } \\
\hline & NWL & WL & Mean & NWL & WL & Mean & NWL & WL & Mean & NWL & WL & Mean & NWL & WL & Mean & NWL & WL & Mean \\
\hline KRL- 3-4 (T) & 67.34 & $\begin{array}{c}\mathbf{5 3 . 5 2} \\
(\mathbf{2 0 . 5 2})\end{array}$ & 60.43 & 89.63 & $\begin{array}{c}78.34 \\
(12.60)\end{array}$ & 83.99 & NWL & WL & Mean & NWL & WL & Mean & 2.90 & $\begin{array}{c}2.61 \\
(10.00)\end{array}$ & 2.76 & 2.96 & $\begin{array}{c}2.86 \\
(3.38)\end{array}$ & 2.91 \\
\hline KRL -99 (T) & 69.66 & $\begin{array}{c}49.62 \\
(28.77)\end{array}$ & 59.64 & 86.10 & $\begin{array}{c}70.64 \\
(17.96)\end{array}$ & 78.37 & 73.60 & $\begin{array}{c}64.60 \\
(12.23)\end{array}$ & 69.10 & 78.60 & $\begin{array}{c}70.34 \\
(10.51)\end{array}$ & 74.47 & 2.81 & $\begin{array}{c}2.64 \\
(6.05)\end{array}$ & 2.73 & 2.89 & $\begin{array}{c}2.67 \\
(7.61)\end{array}$ & 2.78 \\
\hline HD-2851 (S) & 58.81 & $\begin{array}{l}41.43 \\
(29.55)\end{array}$ & 50.12 & 76.47 & $\begin{array}{c}60.65 \\
(20.69)\end{array}$ & 68.56 & 74.80 & $\begin{array}{c}60.30 \\
(19.31)\end{array}$ & 67.55 & 83.60 & $\begin{array}{l}71.26 \\
(14.76)\end{array}$ & 77.43 & 2.86 & $\begin{array}{c}2.46 \\
(13.99)\end{array}$ & 2.66 & 2.72 & $\begin{array}{c}2.56 \\
(5.88)\end{array}$ & 2.64 \\
\hline HD-2009 (S) & 61.23 & $\begin{array}{c}37.86 \\
(38.17)\end{array}$ & 49.55 & 71.58 & $\begin{array}{c}\mathbf{5 3 . 2 1} \\
(25.66)\end{array}$ & 62.40 & 69.63 & $\begin{array}{c}52.91 \\
(24.01)\end{array}$ & 61.27 & 74.60 & $\begin{array}{c}\mathbf{5 9 . 5 0} \\
(\mathbf{2 0 . 2 4})\end{array}$ & 67.05 & 2.89 & $\begin{array}{c}2.32 \\
(19.72)\end{array}$ & 2.60 & 2.69 & $\begin{array}{l}2.51 \\
(6.69)\end{array}$ & 2.60 \\
\hline \multirow[t]{2}{*}{ Mean } & 64.26 & 45.61 & - & 80.95 & 65.71 & - & 68.53 & $\begin{array}{c}50.36 \\
(26.51)\end{array}$ & 59.45 & 70.50 & $\begin{array}{c}56.26 \\
(20.20)\end{array}$ & 63.38 & 2.87 & 2.51 & - & 2.81 & 2.65 & - \\
\hline & $\mathbf{V}$ & WL & VxWL & $\mathbf{v}$ & WL & VxWL & 71.64 & 57.04 & - & 76.83 & 64.34 & - & $\mathbf{V}$ & WL & VxWL & $\mathbf{V}$ & WL & VxWL \\
\hline SEm \pm & 1.14 & 0.81 & 1.61 & 1.51 & 1.07 & 2.14 & $\mathbf{v}$ & WL & VxWL & $\mathbf{V}$ & WL & VxWL & 0.05 & 0.04 & 0.08 & 0.06 & 0.04 & 0.08 \\
\hline CD at $5 \%$ & 3.42 & 2.42 & NS & 4.54 & 3.21 & NS & 1.46 & 1.03 & 2.06 & 1.45 & 1.02 & 2.05 & NS & 0.12 & NS & 0.17 & 0.12 & NS \\
\hline
\end{tabular}


Table.3 Effect of water logging on yield attributes components of wheat varieties under sodic soil

\begin{tabular}{|c|c|c|c|c|c|c|c|c|c|c|c|c|c|c|c|c|c|c|c|c|c|c|c|c|}
\hline \multirow{2}{*}{ Varieties } & \multicolumn{3}{|c|}{$\begin{array}{l}\text { Test Weight } \\
(1000 \text { (grains)) }\end{array}$} & \multicolumn{3}{|c|}{ Grains yield plant ${ }^{-1}(\mathrm{~g})$} & \multicolumn{3}{|c|}{ Biological yield plant ${ }^{-1}$} & \multicolumn{3}{|c|}{$\begin{array}{c}\text { Harvest } \\
\text { index }(\%)\end{array}$} & \multicolumn{3}{|c|}{ EBT plant ${ }^{-1}$} & \multicolumn{3}{|c|}{ No. of ear plant ${ }^{-1}$} & \multicolumn{3}{|c|}{ Ear length(cm) } & \multicolumn{3}{|c|}{ No. of grains ear ${ }^{-1}$} \\
\hline & NWL & WL & Mean & NWL & WL & Mean & NWL & WL & Mean & NWL & WL & Mean & NWL & WL & Mean & NWL & WL & Mean & NWL & WL & Mean & NWL & WL & Mean \\
\hline $\begin{array}{l}\text { KRL- 3- } \\
\text { 4(T) }\end{array}$ & 36.28 & $\begin{array}{r}32.29 \\
(11.00)\end{array}$ & 34.29 & 1.55 & $\begin{array}{c}1.21 \\
(21.94)\end{array}$ & 1.38 & 4.00 & $\begin{array}{c}3.20 \\
(20.00)\end{array}$ & 3.60 & 38.86 & $\begin{array}{l}37.91 \\
(2.44)\end{array}$ & 38.39 & 1.97 & $\begin{array}{c}1.72 \\
(12.69)\end{array}$ & 1.84 & 1.97 & $\begin{array}{c}1.72 \\
(12.69)\end{array}$ & 1.84 & 8.86 & $\begin{array}{c}8.36 \\
(5.64)\end{array}$ & 8.61 & 31.67 & $\begin{array}{c}28.30 \\
(10.64)\end{array}$ & 29.98 \\
\hline KRL -99(T) & 36.65 & $\begin{array}{c}31.70 \\
(13.51)\end{array}$ & 34.18 & 2.23 & $\begin{array}{c}1.63 \\
(26.91)\end{array}$ & 1.93 & 4.27 & $\begin{array}{c}3.39 \\
(20.61)\end{array}$ & 3.83 & 52.24 & $\begin{array}{l}48.10 \\
(7.92)\end{array}$ & 50.17 & 2.67 & $\begin{array}{c}2.23 \\
(16.48)\end{array}$ & 2.45 & 2.67 & $\begin{array}{c}2.23 \\
(19.73)\end{array}$ & 2.45 & 8.58 & $\begin{array}{c}8.01 \\
(6.64)\end{array}$ & 8.30 & 33.00 & $\begin{array}{l}30.13 \\
(8.70)\end{array}$ & 31.57 \\
\hline HD-2851(S) & 36.51 & $\begin{array}{c}29.39 \\
(19.50)\end{array}$ & 32.95 & 1.68 & $\begin{array}{c}0.99 \\
(41.57)\end{array}$ & 1.33 & 4.05 & $\begin{array}{c}2.60 \\
(35.71)\end{array}$ & 3.32 & 41.46 & $\begin{array}{l}38.32 \\
(7.57)\end{array}$ & 39.89 & 1.78 & $\begin{array}{c}1.25 \\
(29.78)\end{array}$ & 1.52 & 1.78 & $\begin{array}{c}1.25 \\
(29.78)\end{array}$ & 1.52 & 8.48 & $\begin{array}{c}7.92 \\
(6.60)\end{array}$ & 8.20 & 31.45 & $\begin{array}{c}27.86 \\
(11.41)\end{array}$ & 29.66 \\
\hline HD-2009(S) & 23.71 & $\begin{array}{c}16.88 \\
(28.51)\end{array}$ & 20.30 & 1.46 & $\begin{array}{c}0.71 \\
(51.37)\end{array}$ & 1.08 & 3.78 & $\begin{array}{c}1.99 \\
(47.35)\end{array}$ & 2.88 & 39.15 & $\begin{array}{l}36.04 \\
(7.94)\end{array}$ & 37.59 & 1.66 & $\begin{array}{c}1.12 \\
(32.53)\end{array}$ & 1.39 & 1.66 & $\begin{array}{c}1.12 \\
(32.53)\end{array}$ & 1.39 & 9.74 & $\begin{array}{c}9.06 \\
(6.98)\end{array}$ & 9.40 & 31.56 & $\begin{array}{c}25.72 \\
(18.50)\end{array}$ & 28.64 \\
\hline \multirow[t]{2}{*}{ Mean } & 33.29 & 27.57 & - & 1.73 & 1.14 & - & 4.02 & 2.79 & - & 42.93 & 40.09 & - & 2.02 & 1.58 & - & 2.02 & 1.58 & - & 8.91 & 8.34 & - & 31.92 & 28.00 & - \\
\hline & V & WL & VxW & V & WL & $\begin{array}{l}\text { Vx } \\
\text { W }\end{array}$ & $\mathbf{v}$ & WL & VxW & $\mathbf{v}$ & WL & $\mathbf{V x W}$ & $\mathbf{v}$ & WL & VxW & V & WL & VxW & V & WL & VxW & V & WL & $\mathbf{V x W}$ \\
\hline $\operatorname{SEm} \pm$ & 0.63 & 0.45 & 0.89 & 0.03 & 0.02 & 0.04 & 0.07 & 0.05 & 0.10 & 0.66 & 0.47 & 0.93 & 0.09 & 0.06 & 0.13 & 0.09 & 0.06 & 0.13 & 0.13 & 0.09 & 0.19 & 0.66 & 0.47 & 0.93 \\
\hline CD at $5 \%$ & 1.89 & 1.34 & NS & 0.09 & 0.06 & 0.13 & 0.21 & 0.15 & 0.30 & 1.98 & 1.40 & 2.80 & 0.27 & 0.19 & NS & 0.27 & 0.19 & NS & 0.40 & 0.28 & NS & 1.98 & 1.40 & NS \\
\hline
\end{tabular}

Table.4 Effect of water logging on $\mathrm{Na}, \mathrm{K}, \mathrm{Ca}, \mathrm{Fe}, \mathrm{Al}$ and $\mathrm{Mn}$ content in leaf (ppm) of wheat varieties under sodic soil at end of water logging

\begin{tabular}{|c|c|c|c|c|c|c|c|c|c|c|c|c|c|c|c|c|c|c|}
\hline \multirow[t]{2}{*}{ Varieties } & \multicolumn{3}{|c|}{$\mathrm{Na}$} & \multicolumn{3}{|c|}{$\mathbf{K}$} & \multicolumn{3}{|c|}{$\mathrm{Ca}$} & \multicolumn{3}{|c|}{$\mathrm{Fe}$} & \multicolumn{3}{|c|}{ Al } & \multicolumn{3}{|c|}{ Mn } \\
\hline & NWL & WL & Mean & NWL & WL & Mean & NWL & WL & Mean & NWL & WL & Mean & NWL & WL & Mean & NWL & WL & Mean \\
\hline $\begin{array}{l}\text { KRL- 3- } \\
(T)\end{array}$ & 4692.67 & 9833.0 & 7262.8 & 33667.0 & 7900.0 & 20783.5 & 4067.0 & 3823.0 & 3945.0 & 128.0 & 740.0 & 434.0 & 40.0 & 475.0 & 257.5 & 30.0 & 51.0 & 40.5 \\
\hline $\begin{array}{l}\text { KRL - } \\
\text { 99(T) }\end{array}$ & 4533.00 & 9500.0 & 7016.5 & 38000.0 & 6500.0 & 22250.0 & 4125.0 & 3945.0 & 4035.00 & 135.0 & 652.0 & 393.5 & 44.0 & 492.0 & 268.0 & 27.0 & $\mathbf{5 8 . 0}$ & 42.5 \\
\hline $\begin{array}{l}\text { HD- } \\
\text { 2851(S) }\end{array}$ & 7900.00 & 10100.0 & 9000.0 & 31000.0 & 5500.0 & 18250.0 & 3633.0 & 3401.0 & 3517.0 & 165.0 & 817.0 & 491.0 & 53.0 & 527.0 & 290.0 & 40.0 & 71.0 & 55.5 \\
\hline $\begin{array}{l}\text { HD- } \\
2009 \text { (S) }\end{array}$ & 8300.00 & 10667.0 & 9483.5 & 29312.0 & 5200.0 & 17256.0 & 3501.0 & 3410.0 & 3455.5 & 156.0 & 893.0 & 524.5 & 60.0 & 549.0 & 304.5 & 44.0 & 73.0 & 58.5 \\
\hline Mean & $\begin{array}{c}6356.42 \\
\mathrm{~V}\end{array}$ & $\begin{array}{c}10025.0 \\
\text { WL }\end{array}$ & $\begin{array}{c}- \\
\mathbf{V} \times W\end{array}$ & $\begin{array}{c}32994.7 \\
\text { V }\end{array}$ & $\begin{array}{c}6275.0 \\
\text { WL }\end{array}$ & $\begin{array}{c}- \\
\mathbf{V} \times W\end{array}$ & $\begin{array}{c}3831.5 \\
\mathrm{~V}\end{array}$ & $\begin{array}{c}3644.7 \\
\text { WL }\end{array}$ & $\begin{array}{c}- \\
\mathbf{V x W}\end{array}$ & $\begin{array}{c}146.0 \\
\mathrm{~V}\end{array}$ & $\begin{array}{c}775.5 \\
\text { WL }\end{array}$ & $\begin{array}{c}- \\
\text { VxW }\end{array}$ & $\begin{array}{c}49.2 \\
\mathrm{~V}\end{array}$ & $\begin{array}{c}510.7 \\
\text { WL }\end{array}$ & $\begin{array}{c}- \\
\mathbf{V x W}\end{array}$ & $\begin{array}{c}35.2 \\
\mathrm{~V}\end{array}$ & $\begin{array}{l}63.2 \\
\text { WL }\end{array}$ & $\begin{array}{c}- \\
\text { VxW }\end{array}$ \\
\hline SEm \pm & 164.99 & 116.6 & 233.3 & 474.5 & 335.5 & 671.1 & 69.2 & 48.94 & 97.8 & 11.0 & 7.8 & 15.6 & 6.6 & 4.7 & 9.4 & 1.0 & 0.7 & 1.5 \\
\hline CD at $5 \%$ & 494.63 & 349.7 & 699.5 & 1422.8 & 1006.0 & 2012.1 & 207.4 & 146.72 & NS & 33.0 & 23.4 & 46.7 & 20.0 & 14.1 & 28.3 & 3.2 & 2.2 & 4.5 \\
\hline
\end{tabular}


Maximum ear length was observed in wheat variety KRL 3-4 which was at par with KRL 99 and HD 2009 while minimum in HD 2851 under all the water logging treatments. However, extent of decrease was less in tolerant varieties; KRL 3-4 and KRL 99 (5$6 \%$ ) as compared to susceptible varieties; HD 2851 and HD 2009(6-7\%). Mean effect of all varieties showed non significant variation in Ear length $(\mathrm{cm})$ among varieties. Mean effect of water logging treatment showed significant decrease in Ear length $(\mathrm{cm})$ under water logging over non water logging.

Data regarding to the number of grains ear ${ }^{-1}$ was significantly decreased in all the wheat varieties under the water logging treatments. However, the reduction under water logging was more in HD 2009 (18.5) followed by HD $2851(11.4 \%)$ and while minimum reduction was observed in KRL 99 (8.7 \%). Interaction effect was found statistically significant showing variable response of varieties to water logging. Grain yield plant ${ }^{-1}$ was reduced significantly in all wheat varieties under water logging treatments. Maximum and minimum grain yield plant $^{-1}$ was record in KRL 99 and HD 2009 with respect to water logging treatments. Water logging produced higher reduction in HD 2009 (51.4\%) followed by HD 2851 (41.6 \%), while lowest in KRL 3-4 $(21.9 \%)$ in water logging condition (control).

Maximum and minimum Biological yield plant $^{-1}$ was record in KRL 99 and HD 2851 with respect to water logging treatment. Higher reduction was observed under water logging in HD 2009 (47.3\%) followed by HD 2851 (35.7\%), while lowest in KRL 3-4 (20.0\%) in comparison to the control. Mean effect of all varieties indicated significant variation in biological yield plant $^{-1}$ among varieties. Maximum reduction in test weight was recorded in susceptible variety HD 2009 (28.5\%) followed by HD 2851(19.5\%). while minimum reduction was in highly tolerant varieties KRL 3-4 (11.0\%) and KRL 99(13.5\%).

Harvest index under water logging treatment was lower than control condition irrespective of the variety. Highest harvest index was recorded in KRL 99 as compared to other varieties while lowest was in KRL 3-4. Reduction was more in susceptible variety HD 2009 (7.94 \%) followed by tolerant varieties KRL 3-4 (2.44\%), in comparison to the control. Similar results have also been reported by Prasad et al., (2004), Savita et al., 2004 and Neog et al., 2002; Sharma et al., 2005 a).

\section{Mineral analysis}

Sodium content (Table 4) in leaf by and large increased with increasing water logging treatments. Maximum and minimum sodium content in leaf was observed in HD 2009 and KRL 99 in all the water logging treatments. Under water logging condition maximum sodium content was obtained in HD 2009 $(10667.0 \mathrm{ppm})$ followed by HD 2851 (10100.0 ppm) while minimum in KRL 99 (9500.0 ppm) in comparison to control. Highest potassium content was observed in wheat variety KRL 3-4 while lowest in HD 2009 in comparison to control. However, greater adverse effect was observed with combined stress indicating that plants capabilities to recover from water logging stress at early growth stage could not be completely restored even until vegetative stage. Highest Calcium content was observed in wheat variety KRL 99 while lowest in HD 2851 in comparison to control. Mean effect of all varieties indicate non significant variation in Calcium content among varieties. Mean effect of water logging treatment showed non significant decrease in Calcium content over non water logging. Maximum and minimum sodium content in leaf was observed in HD 2009 and KRL 99 in 
all the water logging treatments. Under water logging condition maximum increment was obtained in HD 2009 (893.0 ppm) followed by HD $2851(817.0 \mathrm{ppm})$ while minimum in KRL 99 (652.0 ppm) in comparison to control under all the water logging treatments. Aluminum content in leaf by and large increased with increasing water logging treatments. Maximum and minimum sodium content in leaf was observed in HD 2009 and KRL 3-4 in all the water logging treatments. Under water logging condition maximum increment was obtained in HD 2009 (549.0 ppm) followed by HD 2851 (527.0 ppm) while minimum in KRL 3-4 (475.0ppm) in comparison to control under all the water logging treatment.

Maximum and minimum Manganese content in leaf was observed in HD 2009 and KRL 34 in all the water logging treatments. Under water logging condition maximum increment was obtained in HD 2009 (73.0 ppm) followed by HD 2851 (71.0 ppm) while minimum in KRL 3-4 (51.0 ppm) in comparison to control under all the water logging treatments. Similar findings were also reported by Stieger and Feller 1994, Setter and Water 2003, Sharma et al., (2005 a) in pigeon pea, Sairam (2009), Khabaz-Saberi et al., (2012),

In conclusion water logging adversely affects all the wheat varieties but KRL 99 and KRL 3-4 showed relatively low reduction in total chlorophyll, and Total soluble carbohydrate under water logging condition while maximum in HD 2009 HD 2851.Waterlogging of wheat, irrespective of the physiological stage of crop development, reduced days to $50 \%$ flowering and yield attributes but the crop was more susceptible to water logging even at later stage of their vegetative growth. Mineral $\mathrm{Na}, \mathrm{K}, \mathrm{Ca}, \mathrm{F}_{\mathrm{e}}, \mathrm{Al}$ and $\mathrm{Mn}$ content in leaf were also reduced in all wheat varieties under water logging treatments except sodium. Na content in leaf increased with increasing water logging treatments. It is concluded that water logging at vegetative stage was more harmful irrespective of the wheat varieties. Differential response of wheat varieties to water logging clearly indicate that KRL 99 and KRL 3-4 can be used as parents for future germplasm improvement in wheat of water logging tolerance under sodic soil conditions.

\section{Acknowledgment}

Thanks to my guide Dr. A.H. Khan (Head), Department of Plant Physiology, College of Agriculture, Narendra Deva University of Agriculture and Technology, Kumarganj, Faizabad (U.P.) for his most valuable and inspiring guidance, close supervision, constant encouragement and constructive criticism coupled with valuable suggestions during the course of this investigation and in preparation of this manuscript.

\section{References}

Bao, X. 1997. Study on identification stage and index of water logging tolerance in various wheat genotype (Triticumaestivum L). Acta Agri. Shanghai, 13(2): 32-38.

Baranwal Sarit, Singh, B.B. 2002. Effect of water logging on growth, chlorophylls and saccharides content in maize genotypes. Indian J. Plant Physiol., 7(3): 246-251.

Das, K.K., Sarkar, R.K. 2003. Post flood changes on the status of chlorophyll, carbohydrate and nitrogen content and its association with submergence tolerance in rice. Plant Archiever, 1(12): $15-19$.

Dong, D.F., Luo, B.S., Chen, D.Q. 1998. Comparative study on some physiological characteristics of wheat waterlogged at seedling and booting 
stage. J. Guangxi Agril. Univ., 17(4): 351-355.

Gardner, W.K., Flood, R.G. 1993. Less water logging damage with long season wheats. Cereal Res. Comm., 21: 337 343.

Khabaz-Saberi, H., Barker, S.J., Rengel, Z. 2012. Tolerance to ion toxicities enhances wheat (Triticumaestivum L.) grain yield in waterlogged acidic soils. Plant and Soil, 354(1/2): 371-381. 32.

Lin Ji, Xiang Li, Xiao Yu, Zhang Zhao, Jun Yu, Xing Yang, Gao Zhan, Wu Wang Ying, Wang Jun, Feng Li, Zhuo Lin Mu, Chun Sheng. 2012. Salinityalkalinity tolerance in wheat: seed germination, early seedling growth, ion relations and solute accumulation. African J. Agri. Res., 7(3): 467-474.

Neog, B., Gogoi, Nirmali, Baruah K.K. 2002. Morpho-physiological changes associated with water logging in rice (Oryza sativa L.) Indian J. Agri. Sci., 72(7): 404-407.

Pattanagul Wattana, Thitisaksakul Maysaya. 2008. Effect of salinity stress on growth and carbohydrate metabolism in three rice (oryza sativa L.) cultivars differing in salinity tolerance. Indian J. Exp. Biol., Vol. 46, pp.736-742.

Prasad Shambhoo, Ram, P.C., Singh Uma. 2004. Effect of waterlogging duration on chlorophyll content, nitrate reductase activity, soluble sugar and grain yield of maize. Ann. Plant Physiol., 18(1): 1-5.

Rai Rajesh Kumar, Srivastava, J.P., Shahi, J.P. 2004. Effect of water logging on some biochemical parameters during early growth stages of maize. Indian $J$. Plant Physiol., 9(1): 65-68.

Ram, P.C., Singh, B.B., A.K. Ram, P. Singh, P.N. Singh, H.P. Boamfa, I. Harren, F. Santosa, E. Jackson, M.B. Setter, T.L. Reuss, J. Wade, L.J. Singh, V.P., Singh, R.K. 2002. Physiological basis of submergence tolerance. Prospects for germplasm improvement through marker aided breeding. Field Crop Res., 76: 131-152.

Sairam, R.K. 2009. Salinity stress tolerance in crop plants. National conference on Frontiers in Plant Physiology towards sustainable agriculture, 5-7 Nov., Jorhat, Assam.

Samad, A., Meisner, C.A., Saifuzzaman, M., Van Ginkel, M. 2001. Waterlogging tolerance. In Application of Physiology in Wheat Breeding. Eds. M.P. Reynolds, J.I. Ortiz-Monasterio and A. McNab. pp. 136-144. CIMMYT, Mexico.

Savita, U.S., Rathore, T.R., Mishra, H.S. 2004. Response of some maize genotypes to temporary waterlogging. $J$. Plant Biol., 31(1): 29-36.

Sayre, K.D., van Ginkel, M., Rajaram, S., Ortiz-Monasterio, I. 1994. Tolerance to water-logging losses in spring bread wheat: effect of time of onset on expression. In: Annual Wheat Newsletter No. 40. Colorado State University, Fort Collins, 165-171.

Setter, T.L. 2006. Preliminary report on water logging tolerance of wheat in India and Australia. ACIAR Project CS1/1996/025. Department of Agriculture and Food, Western Australia, S. Perth; and Australian Centre for International Agricultural Research, Canberra, ACT.

Sharma, D.P., Singh, M.P., Gupta, S.K., Sharma, N.L. 2005a. Response of pigeonpea to short-term water stagnation in a moderately sodic soil under field conditions. J. Indian Soc. Soil Sci., 53(2): 243-248.

Sharma, D.P., Swarup, A. 1988. Effect of short-term flooding on growth, yield and mineral composition of wheat on sodic soil under field conditions. Plant Soil, 107:137-143. doi:10.1007/ BF02371555. 
Sharma, S.K., Praveen, K.S., Setter, T.L., Singh, K.N. 2004. Genetic diversity in waterlogging tolerance of wheat genotypes in neutral and sodic soil at the seed germination stage.In Proceedings of International Conference on Sodicity, Lucknow, pp. 264-267.

Shimada, S., Kokubun, M., Matsui, S. 1995. Effects of water table on physiological traits and yield of soybean I. Effects of water table and rainfall on leaf chlorophyll content, root growth and yield. Japanese J. Crop Sci., 64: 105112.

Singh Dhananjaya, Sharma, P.C. 2010. Effect of $\mathrm{NaCl}$ stress on photosynthesis characteristics and antioxidant enzymes in rice cultivars. Indian J. Plant Physiol., Vol. 15, No.3 (N.S.)PP. 255258.
Singh, P.N., Ram, P.C. Singh, Amit, Singh, B.B. 2005. Effect of seedling age on submergence tolerance of rainfed lowland rice. Ann. Plant. Physiol., 19(1): 22-26.

Srivastava Rashmi, Chaturvedi, G.S., Singh Manish Kumar, Singh Amit. 2004. Effect of submergence on plant growth parameters of soybean. Ann. Plant Physiol., 18(2): 116-120.

Stigger, P.A., U. Feller. 1994. Nutrient accumulation and translocation in maturing wheat plant grown own water logging soil. Plant and Soil, 160(1): 8796.

Watson, E.R., Lapins, P., Barron, R.J.W. 1976. Effect of water logging on the growth, grain and straw yield of wheat, barley and oats. Aust. J. Exp. Agric. Anim. Husb., 16: 114-122.

\section{How to cite this article:}

Ompal Singh, S.P. Singh, Raj Bahadur, Mani Ram, Prashansha Singh and Sunil Prajapati. 2017. Physiological Basis of Water Logging Tolerance in Wheat at Vegetative Stage Under Sodic Soil. Int.J.Curr.Microbiol.App.Sci. 6(4): 1993-2004.

doi: https://doi.org/10.20546/ijcmas.2017.604.236 